\title{
Refractory Myxoid Liposarcoma
}

National Cancer Institute

\section{Source}

National Cancer Institute. Refractory Myxoid Liposarcoma. NCI Thesaurus. Code C148297.

Myxoid liposarcoma that does not respond to treatment. 